\title{
En attendant les gènes, un nouveau test d'aide au conseil génétique pour les syndromes de Prader-Willi et d'Angelman
}

La région 15q11-q13 est soumise à empreinte parentale, avec spécificité maternelle ou paternelle selon les gènes concernés [1]. Sous empreinte, un seul des deux allèles s'exprime, l'autre étant méthylé, avec asynchronisme de réplication. Quand la région est délétée, les allèles devant s'exprimer étant absents, il en résulte des anomalies phénotypiques, différentes selon l'origine parentale du chromosome porteur de la délétion: syndrome de Prader Willi (PWS) quand celle-ci est sur le chromosome paternel, syndrome d'Angelman (AS) quand elle est sur le chromosome maternel $(\mathrm{m} / \mathrm{s}$ $1991 n^{\circ} 9$, vol. 7, p. 974.). Bien que la région ait été soigneusement explorée, on ne sait pas encore avec certitude quels sont les gènes responsables de l'un et de l'autre de ces deux syndromes, mais on dispose de nombreuses sondes de la région, parmi lesquelles celle du gène $S N R P N$ (qui est un bon candidat pour le PWS) et la sonde D15S9, dont les locus sont situés dans la plus petite région délétée commune correspondant à $70 \%$ des cas de PWS et d'AS. Marquées en fluorescence et grâce à la technique de FISH (hybridation in situ en fluorescence), elles permettent de dépister les délétions. Mais celles-ci n'expliquent évidemment pas toutes les anomalies génétiques de ce segment d'ADN soumis à l'empreinte. Les disomies uniparentales (UPD) du chromosome 15 ont les mêmes conséquences : la disomie 15 maternelle étant responsable de PWS (25\% des cas) par absence de l'allèle paternel qui, probablement, est le seul à s'exprimer (ce qui est le cas du gène $S N R P N$ ), et la disomie 15 paternelle responsable d'AS (5\% des cas), par absence de l'allèle maternel. Quant aux autres malades, ils ont bien reçu un chromosome 15 de chacun de leurs parents, mais cette disomie biparentale (BPD), qui est la situation normale, s'accompagne d'une anomalie de la méthylation et de mutations dans la séquence de l'ADN située en amont du gène $S N R P N$ dans les PWS. Reste enfin un très petit nombre de cas de PWS et d'AS (moins de $5 \%$ en tout) avec disomie biparentale sans délétion décelable ni anomalie de la méthylation. Or, et c'est bien là le problème, si le risque de récurrence est quasiment nul dans les syndromes dus à des délétions ou à des disomies uniparentales (elles surviennent de novo), ce risque est de $50 \%$ dans les familles de PWS et d'AS avec disomie biparentale. En l'absence de délétion, comment faire pour distinguer les cas dus à une UPD, qui sont les plus nombreux ? Il fallait trouver un test, facile à réaliser, peu coûteux, et ne nécessitant pas l'analyse moléculaire de la région 15q11-q13 des deux parents pour pouvoir rassurer ces couples ayant déjà un enfant atteint de PWS ou d'AS, souhaitant une grossesse et n'ayant pas plus de risque de récurrence que la population générale. Une équipe américaine vient de mettre au point une technique qui semble remplir les conditions précitées [2]. Elle repose sur cet asynchronisme de réplication qui est normal entre les deux allèles reçus des deux parents et soumis à empreinte parentale. En utilisant les mêmes sondes marquées en fluorescence, $S N R P N$ et $D 15 S 9$, avec la technique de FISH, mais cette fois sur noyaux en interphase, on peut suivre les phases du cycle cellulaire, G1, S et G2. En G1, on voit deux signaux lumineux séparés dans le noyau, correspondant aux deux allèles non encore répliqués. En G2, les deux signaux se sont dédoublés, et deux paires de points lumineux sont visibles. Dans la phase S, dite G1/G2, un seul des deux allèles est répliqué, l'image est asymétrique avec un point isolé et un signal double. Dans les conditions de l'étude, le pourcentage des noyaux dans les trois phases du cycle est constant : environ $66 \%$ en G1, $7 \%$ en G2, et $27 \%$ en phase G1/G2. Cela, bien entendu, si les chromosomes homologues proviennent de chacun des deux parents, ce qui est le cas chez les témoins et chez les malades avec disomie biparentale. Quant aux malades avec UPD, on aurait pu s'attendre à une absence d'image G1/G2 puisqu'il n'y a pas d'asynchronisme de réplication quand les homologues proviennent du même parent. Mais, pour des causes stochastiques, on observe environ $8 \%$ de noyaux en G1/G2. Toutefois, l'absence de chevauchement entre les BPD avec $27 \%$ de G1/G2 et des UPD avec $7 \%$ permet de les distinguer nettement. Cette méthode étant quantitative, il est souhaitable que d'autres équipes corroborent ces résultats avant de généraliser ce test rapide, peu onéreux et qui se pratique sur les préparations mêmes ayant servi à éliminer une délétion. Il pourrait éclairer le conseil génétique dans $95 \%$ de familles avec PWS et $75 \%$ des familles avec AS. En attendant, la technique est dès maintenant utilisable pour l'exploration des autres régions soumises à empreinte parentale.

S.G.

1. Paldi A, Jami J. Éléments chromosomiques contrôlant l'empreinte parentale des gènes. médecine/sciences $1996 ; 12$ : 189-91.

2. White LM, Rogan PK, Nicholls RD, Wu BL, Korf B, Knoll JHM. Allele-specific replication of 15q11q13 loci : a diagnostic test for detection of uniparental disomy. Am J Hum Genet 1996 ; 59 : 423-30. 